\title{
Relation of Hand Enthesophytes with Knee Enthesopathy: Is Osteoarthritis Related to a Systemic Enthesopathy?
}

\author{
NADIA GIBSON, ALI GUERMAZI, MARGARET CLANCY, JINGBO NIU, PETER GRAYSON, PIRAN ALIABADI, \\ FRANK ROEMER, and DAVID T. FELSON
}

\begin{abstract}
Objective. Enthesopathy has been reported as a feature of osteoarthritis (OA) in the distal interphalangeal (DIP) joints. We previously reported that central bone marrow lesions (BML) on magnetic resonance imaging (MRI) scans are associated with OA. In this study, we evaluated whether hand and knee enthesopathy were related.

Methods. We studied knee and hand radiographs of subjects from the Framingham Osteoarthritis Study. Subjects seen in 2002-2005 had bilateral posteroanterior hand radiographs, weight-bearing knee radiographs, and knee MRI scans. Hand radiographs were read for enthesophytes at the juxtaarticular nonsynovial areas of metacarpophalangeal (MCP), proximal interphalangeal (PIP), and DIP joints, and midshafts of the phalanges. We selected 100 cases of knees with central BML and 100 matched controls. Conditional logistic regression was used to assess associations.

Results. Subjects with enthesophytes of at least 1 score $\geq 2$ at DIP, PIP, and/or MCP were not more likely to have central knee BML (OR 0.49,95\% CI 0.17-1.40) than those without enthesophytes. Similarly, having at least 1 score $\geq 2$ on the shafts was not significantly associated with having a central knee BML (OR 0.59, 95\% CI 0.23-1.51). Adjustment for the presence of diabetes mellitus did not affect these results, but there was an increased prevalence of diabetes in those with hand enthesophytes (OR 3.09, 95\% 1.29-7.40, enthesophyte score $\geq 2$ ).

Conclusion. We found no increase in the prevalence of hand enthesophytes among persons with central knee BML on their knee MRI scans. This provides evidence against a systemic enthesopathic disorder in association with knee OA. (First Release Dec 15 2011; J Rheumatol 2012;39:359-64; doi:10.3899/jrheum.110718)
\end{abstract}

\section{Key Indexing Terms:} OSTEOARTHRITIS

\section{ENTHESOPHYTE}

Osteoarthritis $(\mathrm{OA})$ is a multifactorial process resulting in varying degrees of joint failure. It has been suggested that in some presentations OA may be a generalized process with a polyarticular involvement ${ }^{1}$. Although this polyarticular subtype of OA is thought to exist and has been termed generalized $\mathrm{OA}$, it has not been clearly defined. Joints frequently affected in persons with generalized OA include the knees and hands ${ }^{2,3,4}$.

Entheses are the insertion sites of ligaments, tendons, or articular capsule onto bone. Bony spurs at these sites are called enthesophytes. Enthesophytes accompany inflammato-

From the Departments of Clinical Epidemiology, Rheumatology and Radiology, Boston University Medical Center, and the Department of Radiology, Brigham and Women's Hospital, Boston, Massachusetts, USA. Supported by NIH AR47785.

N. Gibson, MD; A. Guermazi, MD; M. Clancy, MPH; J. Niu, DSc; P. Grayson, MD, Boston University Medical Center; P. Aliabadi, MD, Department of Radiology, Brigham and Women's Hospital; F. Roemer, MD; D.T. Felson, MD, MPH, Boston University Medical Center. Address correspondence to Dr. D.T. Felson, Boston University Medical Center, 650 Albany Street, X Building, Suite 200, Boston, MA 02118, USA. E-mail:dfelson@bu.edu

Accepted for publication October 28, 2011.

\section{CENTRAL BONE MARROW LESIONS}

ry disorders, especially the seronegative spondyloarthropathies, but may also accompany degenerative, endocrine, and metabolic disorders ${ }^{5}$. A skeletal survey study found a positive correlation between eburnation and osteophyte and enthesophyte formation ${ }^{6}$, and it was suggested that a proportion of the population can be classified as "bone formers" based on an association of osteophyte and enthesophyte formation ${ }^{7}$. Some problems with this study have been identified ${ }^{8}$. The investigators were unable to accurately characterize the ages of the specimens and it is possible the correlation of enthesophyte and osteophyte formation was more related to their increased prevalence in older subjects. They also did not have information on underlying diseases that could confound findings such as inflammatory arthritis or diffuse idiopathic skeletal hyperostosis (DISH). The effects of these possible confounders are illustrated by the high incidence of wrist involvement - an atypical site for OA $(3.8 \%$ and $11.9 \%$ prevalence of eburnation and osteophyte, respectively, in wrists compared to $4.0 \%$ and $11.0 \%$ in knees).

Tan, et al found that enthesophytes at the insertion sites of collateral ligaments and tendons were a consistent and early feature in the development of hand $\mathrm{OA}^{9}$. Many enthesis sites exist as a component of an "enthesis organ." The term organ 
illustrates the concept of these insertion sites as a composite of various parts that include, in addition to the ligament or tendon insertion, sesamoid or periosteal fibrocartilage, synovium, and possibly a bursa ${ }^{10}$. These complexes serve to dissipate mechanical stress from the insertion site and it is hypothesized there may be a connection between the presence of "enthesis organs" and generalized nodal $\mathrm{OA}^{11}$ as an extension of the findings of Tan, et $a l^{9}$. Anterior and posterior cruciate insertions have been identified as sites where there are enthesis organs, as have digital extensor and flexor tendon insertions and digital collateral ligaments ${ }^{10}$. In the knee, central bone marrow lesions [BML; ill-defined hyperintense areas on T2- or proton density-weighted fat-suppressed magnetic resonance imaging (MRI) at or between the tibial spines or the femoral notch] may represent enthesopathic changes related to insertion sites of the anterior and posterior cruciate ligaments (ACL, PCL; Figure 1). Histologically, these lesions represent areas of necrosis, microcracks, and fibrosis ${ }^{12}$. In an MRI study examining knees with symptomatic OA, 53.8\% of subjects were found to have central BML even in the absence of ACL/PCL tears ${ }^{13}$.

The objectives of our study were to determine if the enthesopathic changes seen by McGonagle and those described in the central regions of the knee were related, suggesting that one subtype of OA may be that of a generalized enthesopathy. To address this issue, we investigated whether central bone marrow lesions on knee MRI were associated with hand enthesopathy.

\section{MATERIALS AND METHODS}

The Framingham Osteoarthritis Study is a population-based cohort study of OA. Our cohort consisted of the offspring cohort subjects of the Framingham Heart Study and a community-based cohort recruited from Framingham, Massachusetts ${ }^{14,15}$. Members of this combined cohort make up the Framingham Osteoarthritis cohort and they were examined between 2002 and 2005.

The community-based cohort was recruited by random-digit dialing and census tract data to provide a representative sample of the Framingham community. Subjects were eligible for inclusion if their age was $>50$ years. Subjects were excluded if they had undergone bilateral knee replacements or had a history of rheumatoid arthritis (RA). The history of RA was determined by a validated survey instrument ${ }^{16}$. We did not exclude subjects using ambulatory-assistive devices.

Bilateral posteroanterior (PA) plain hand radiographs and bilateral weight-bearing PA plain knee radiographs in fixed-flexion were obtained from subjects who agreed to participate following recruitment. All participants from the community cohort underwent MRI of knees. To limit burden to respondents in the offspring cohort, knee MRI scans were performed only on those who replied "yes" to the question "In the past 30 days, have you had any pain, aching, or stiffness in either of your knees?"

Radiographs and MRI. Using the fixed-flexion PA radiographs of the knees, tibiofemoral OA was scored by Kellgren-Lawrence (K-L) grade ( 0 to 4$)^{17}$ by an academically based musculoskeletal radiologist. OA was defined as present when the score was $\geq 2$ in a knee.

Hand enthesophytes. The hand radiographs were read for enthesophytes by a rheumatologist (NG) trained before the study by musculoskeletal radiologists (AG, PA) in reading enthesophytes on plain hand radiographs. As the first digit does not project well on plain PA radiographs, enthesophytes on only digits 2 through 5 were scored for both hands of each participant. Enthesophytes were scored on a scale of $0=$ normal, $1=$ mild, $2=$ moderate, and $3=$ severe at each of the midshafts of the proximal, middle, and distal phalanges and juxtaarticular nonsynovial areas of the metacarpophalangeal

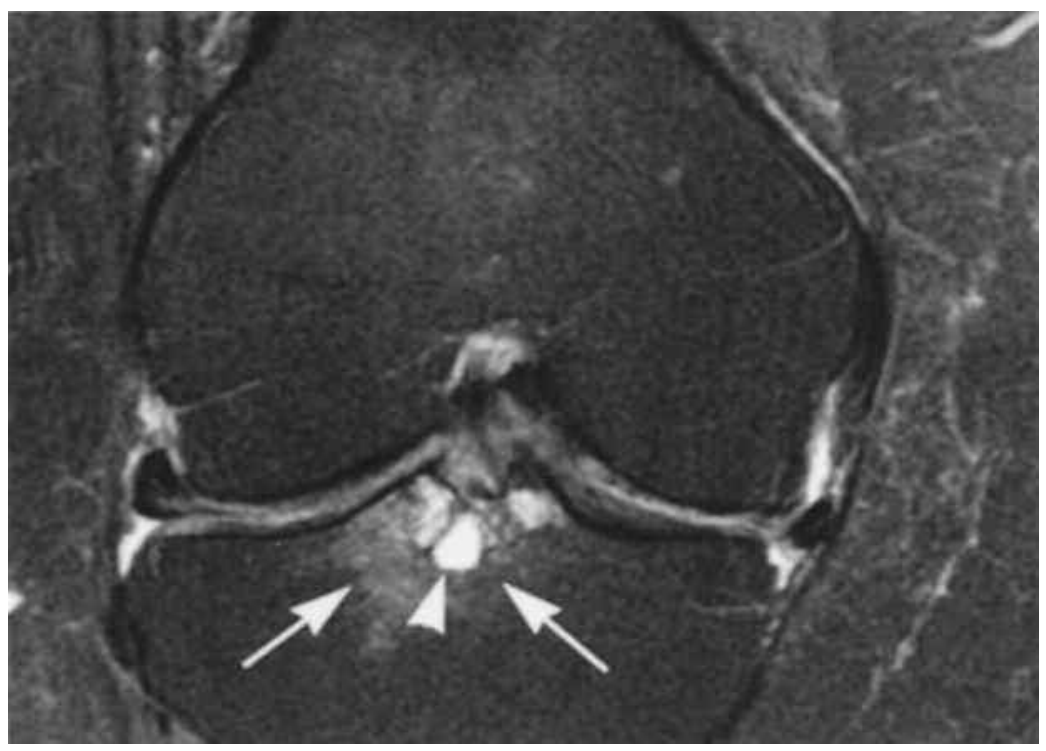

Figure 1. Femoral notch bone marrow lesion (BML). Coronal proton density-weighted fat-suppressed magnetic resonance imaging (MRI) shows grade 3 WORMS (whole-organ MRI score) subchondral bone marrow lesion at the tibial subspinous region (arrows) with a cystic component (arrowhead). Image shows the BML at the tibial insertion of the anterior cruciate ligament. There is medial and lateral meniscal subluxation and partial maceration of the body of the medial meniscus. There is a diffuse thinning of the cartilage of the central medial femur. 
(MCP) and proximal (PIP) and distal interphalangeal (DIP) joints (Figures 2, 3 , and 4), similarly to the grading scheme for osteophytes. When there were $\geq 2$ enthesophytes in the same subregion, we focused on the highest score. Intraobserver agreement was found to be $\mathrm{k}=0.70(\mathrm{p}<0.001)$.

Knee MRI protocols and definitions. MRI scans were obtained using the Signa 1.5 Tesla system (Siemens Healthcare, Erlangen, Germany) using a phased-array knee coil. A device was used to ensure consistent knee positioning by patients. Proton density-weighted (PDw) fat-suppressed images in the sagittal and coronal planes were obtained with the following pulse sequence variables: $\mathrm{TR}=3610 \mathrm{~ms}$, TE $=40 \mathrm{~ms}$, slice thickness $=3.5 \mathrm{~mm}$, and field of view $=14 \mathrm{~cm}$. Central subspinous BML were defined as ill-defined (no defined rim) areas within trabecular bone that appeared hyperintense in PDw fat-suppressed sequences at or between the spinous tibial plateaus or adjacent to the femoral notch. Central subspinous BML were read by AG and 1 other experienced musculoskeletal radiologist (FR). The interrater kappa for BML was $0.67(\mathrm{p}<0.001)$. Enthesopathy at the insertion of the joint capsule and medial and lateral collateral ligaments at the knee was not determined, as it is difficult to accurately assess changes at these areas on MRI.

Selection of cases and controls. Subjects from the Framingham Osteoarthritis Study with central BML grade $\geq 2$ on knee MRI were selected as cases. An equal number of subjects matched for sex and age without BML on knee MRI were chosen as the control group. Hand radiographs from cases and controls were randomly sorted and read blinded to case/control status.

Statistical analysis. Presence of an enthesophyte was classified as a dichotomous outcome, 1 indicating presence of 1 or more grade 2 enthesophytes, and 0 the absence of enthesophytes by this definition. The data were analyzed by conditional logistic regression to assess for the association of BML and presence of enthesophytes in the hand. Crude scores were adjusted for maximal $\mathrm{K}-\mathrm{L}$ grade in the knee joint per subject.

In subanalyses, we limited our cases and controls to subjects with knee OA with K-L grade $\geq 2$ with the same definition of case versus control as pre-

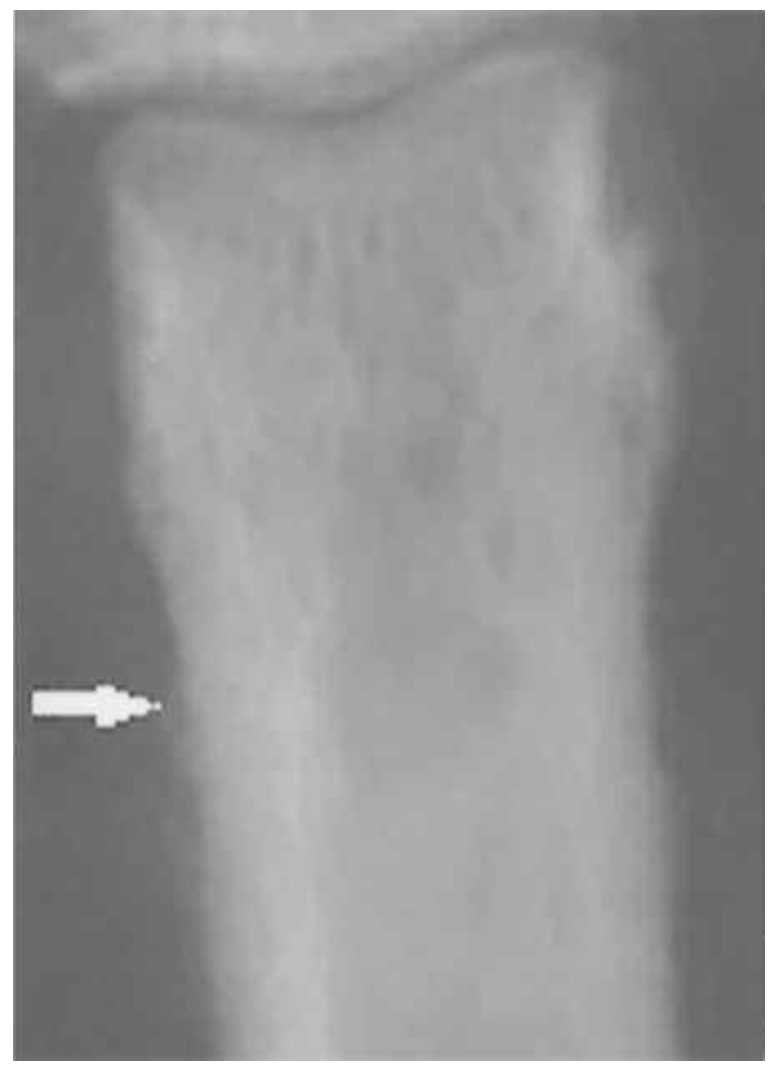

Figure 2. Grade 1 proximal shaft enthesophyte.

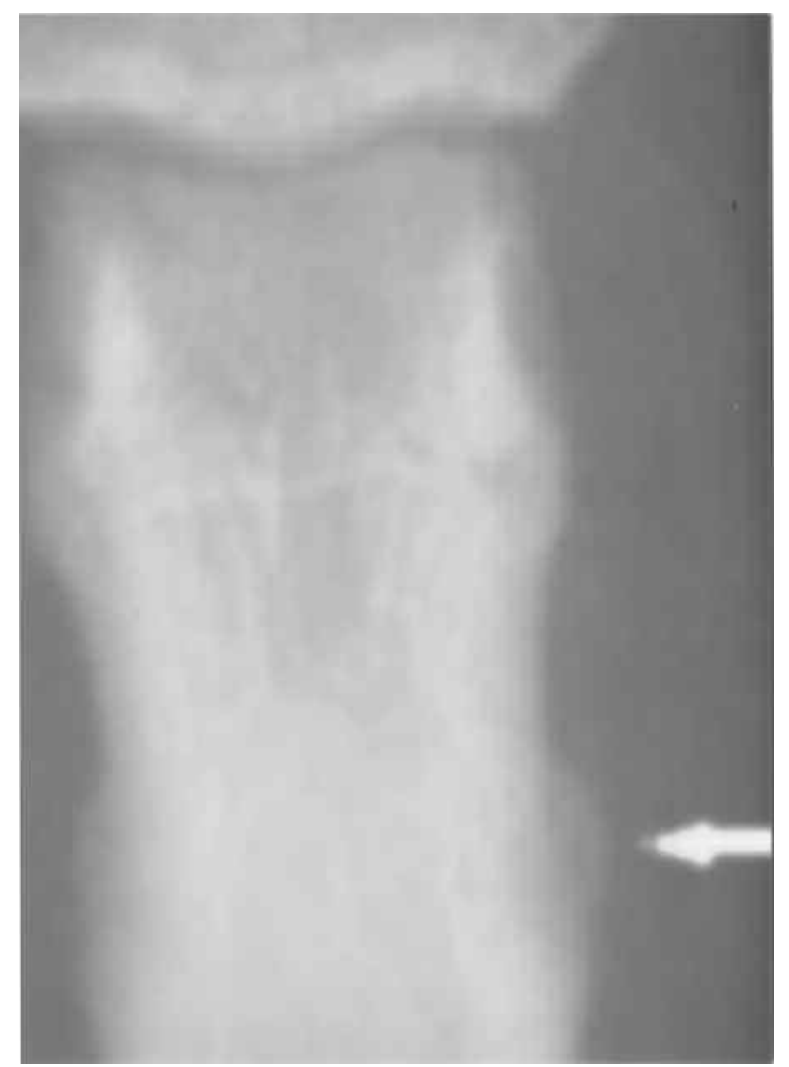

Figure 3. Grade 2 proximal midshaft enthesophyte.

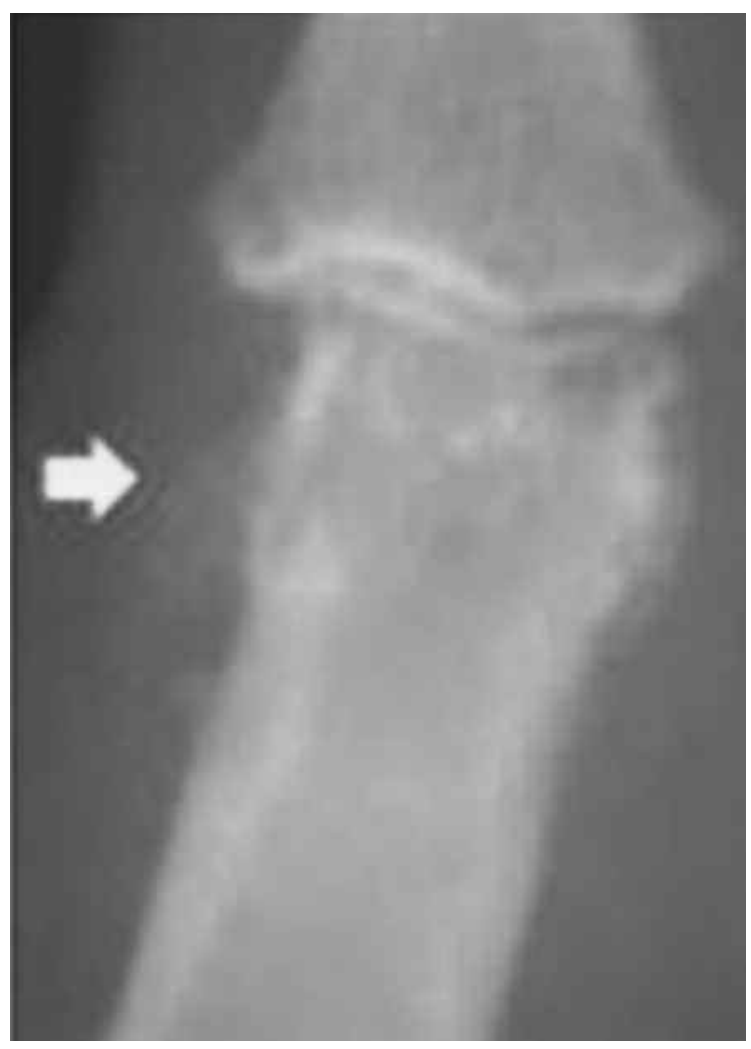

Figure 4. Grade 3 proximal interphalangeal enthesophyte. 
viously, i.e., subjects with and without central subspinous BML, respectively. As this was a subgroup of the initial cohort, it was not matched for age and sex. A logistic regression analysis to measure the relation between hand enthesophytes and BML was conducted in this subgroup. In additional analyses to ensure diabetes was not confounding our findings, we controlled for the presence of diabetes mellitus (DM; defined by subject's positive response to question of being given a prior diagnosis of DM or high blood glucose). We also performed a logistic regression among the BML cases and controls separately, assessing the association of DM and hand enthesophytes. We present $95 \%$ confidence bounds and 2-sided p values as part of our results, but note that we were especially interested in whether there was an increased (not decreased) odds of hand enthesopathy in those with central BML in the knee. Because of this a priori question, we focused particularly on the upper bound of the $95 \%$ CI, testing the relation of hand enthesophytes to knee BML. With 95\% likelihood, the association is not stronger than this value.

\section{RESULTS}

There were 105 pairs of cases and controls - case being defined as having central BML on knee MRI. Among these 105 pairs, 5 did not have hand enthesophytes among either cases or controls; as they were not informative in terms of the association of interest, they were excluded from the analysis. There were 100 pairs left for analysis, 33 from the Framingham offspring cohort and 67 from the community cohort. Of these, there were 50 women and 50 men. The mean age of cases was $68.5 \pm$ SD 8.8 years and of controls $68.2 \pm$ SD 8.5 years (Table 1 ).

Hand enthesophytes. In the basic cohort, 5 out of 100 case subjects had at least 1 enthesophyte score $\geq 2$ at DIP, compared to 6 controls (Table 2). Twenty cases and 23 controls had at least 1 enthesophyte at the DIP, PIP, or MCP joints. Those with enthesophytes with a score $\geq 2$ were less likely to have central BML of the knee, but not statistically significantly (OR 0.49, 95\% CI 0.17-1.40), a finding not changed when we controlled for the presence of diabetes. Having at least 1 enthesophyte score $\geq 2$ at the distal, middle, or proximal shafts was not associated with having central knee BML (OR 0.59, $95 \%$ CI $0.23-1.51$ ). Twenty-four out of 100 case subjects versus 27 of 100 controls were found to have at least 1 enthesophyte score $\geq 2$ at the shaft sites. Considering all hand sites, the number of subjects with at least 1 enthesophyte score $\geq 2$

Table 1. Characteristics of the participants according to the presence or absence of central bone marrow lesions (BML) on knee magnetic resonance imaging.

Among BML Cases, Among BML Controls, $\mathrm{n}=100$ $\mathrm{n}=100$

\begin{tabular}{lcc}
\hline Age, mean (SD), yrs & $69(9)$ & $68(9)$ \\
Body mass index, mean (SD) & $29.7(5)$ & $29(6)$ \\
Maximal K-L grade*, \% & & \\
$0-1$ & 33 & 75 \\
2 & 15 & 10 \\
3 & 21 & 12 \\
4 & 31 & 3 \\
Diabetes, \% & 11 & 14 \\
\hline
\end{tabular}

* One control had K-L grade missing. K-L: Kellgren-Lawrence grade. was 38 in the cases and 42 in controls (OR 0.57, 95\% CI 0.26-1.27). Further adjustment for diabetes did not change this finding. Analyses using enthesophyte score $\geq 1$ yielded similar results. In analyses adjusting for the presence of diabetes and examining all ways in which enthesophytes might be counted, the upper bound of the $95 \%$ CI was 1.30 , suggesting that our data are incompatible with a greater than $30 \%$ increase in the odds of enthesophytes in persons with central BML.

Cohort limited to subjects with $K-L$ grade $\geq 2$. Sixty-seven subjects with central BML and 26 without central BML from the original cohort had maximal K-L grade $\geq 2$. Although not matched, there was no substantial difference in age or BMI between these subsets of cases and controls. These analyses revealed results similar to those of the larger case-control group and did not produce statistically significant results.

Analysis of association with DM. Examining cases and controls collectively and comparing those with DM to those without, there was a statistically significant increased risk of DM among subjects with enthesopathy at the distal, middle, and proximal shafts compared to those without (OR 3.91, 95\% CI $1.65-9.28$ for enthesophyte score $\geq 2$ ). However, there was no significantly increased prevalence of DM in subjects with DIP, PIP, and MCP enthesophytes (OR 1.17 95\% CI 0.44-3.16).

This association of DM with enthesopathy was strong especially in controls. Among controls, those with enthesophyte scores $\geq 2$ at the distal, middle, or proximal shafts were more likely to have DM with statistical significance (OR 4.77, 95\% CI 1.48-15.44). Similarly, when all sites were considered there was a statistically significant association of hand enthesophytes with DM (OR 6.62, 95\% CI 1.71-25.55).

\section{DISCUSSION}

We examined if associations existed between hand enthesopathy and central BML in knees and investigated their relationship to OA. We found that having central BML in the knee did not increase the risk of having enthesophytes at the various identified areas on hands, and subsequently from our results there was no indication of a relation between hand enthesopathy and central BML of the knees. There was a moderate prevalence of hand enthesophytes among both cases and controls, with a near-equal distribution between capsular (DIP, PIP, MCP) and midshaft enthesophytes. Considering all sites, $38 \%$ of cases and $42 \%$ of controls were found to have enthesophytes. The upper bound of the CI in our analyses usually remained below 1.6, and was often lower than this, suggesting that those with central BML were not at substantially increased risk of hand enthesophytes.

In subanalysis, we restricted cases and controls to subjects with knee OA with K-L grade $\geq 2$. There was a higher percentage of advanced knee OA in the cases; 67/100 with grade $\geq 2$, in contrast to $26 / 100$ in controls. The percentage of subjects with hand enthesophytes among both cases and controls 
Table 2. Association of subspinous bone marrow lesions seen on knee MRI and enthesophytes seen on hand radiographs.

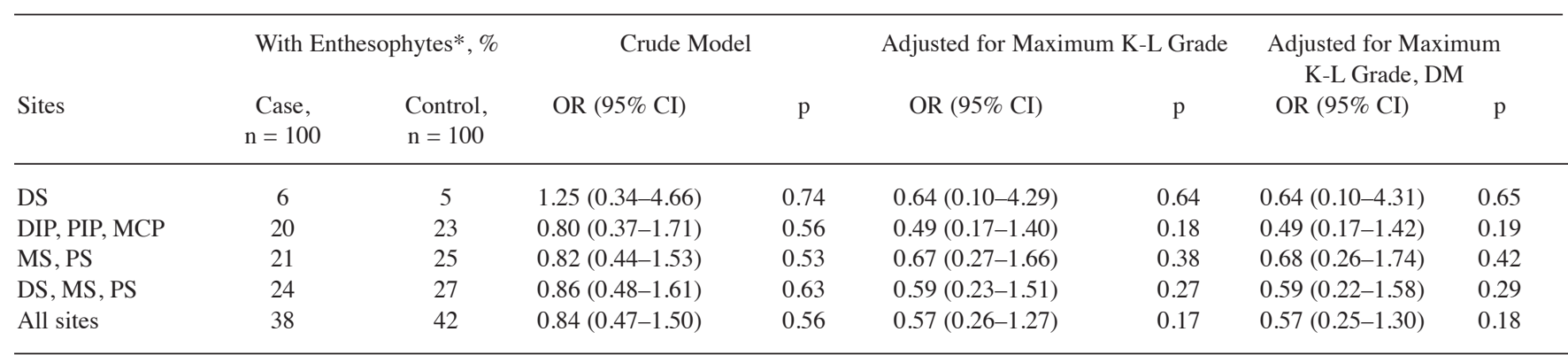

* Percentage of subjects with at least 1 enthesophyte score $\geq 2$ at various sites; $\mathrm{n}=100$ in both cases and controls. DM: diabetes mellitus; DS: distal shaft; DIP: distal interphalangeal joint; PIP: proximal interphalangeal joint; MCP: metacarpophalangeal joint; MS: midshaft; PS: proximal shaft.

was uniformly higher than in the original cohort, although there was no significant difference between cases and controls in this subset. This may be suggestive of a previously noted association of osteophyte and enthesophyte formation, either as a predisposition of these subjects to develop these lesions or related to the increased mean age of this group and the increased prevalence of these findings in older subjects.

A radiographic study previously found a correlation between hand osteophytes and hand midshaft enthesophytes on plain radiographs, but ultimately this correlation was diminished by correction for age and it was proposed that age was the predominant factor that linked these findings ${ }^{18}$. Also, as noted, a radiographic study of knees established a connection between knee central BML and progression of knee $\mathrm{OA}^{13}$. This finding was also supported here with the higher prevalence of advanced OA in the cases. It is possible that these associations represent local events as opposed to systemic processes, such that the primary drivers in the development of knee central subspinous BML are confined to events restricted to that area and are not part of a more generalized process. As suggested by Kalichman, et al, these various types of ectopic bone formation may be outcomes of differing processes although coexisting within the same subject ${ }^{18}$.

We did find a relationship between self-reported DM and hand enthesophytes. This was more evident when assessing midshaft enthesopathy. This is likely explained at least in part by the increased predisposition to DISH in persons with DM, one of the features of which is enthesopathy. In describing the findings on hand radiographs of subjects with DISH in comparison with normal controls and subjects with acromegaly, Littlejohn, et al $^{19}$ found there was an increased prevalence of both capsular and proximal phalanx enthesopathy in the DISH group compared to the controls. All subjects with DISH had proximal phalanx enthesopathy and there was more likely to be a marked degree of enthesopathy as compared to capsular enthesopathy (comparable to DIP, PIP, and MCP enthesophytes in our study). Confirmation in our data of the known relationship of diabetes with enthesopathy validates our enthesopathy hand readings.

There were some limitations in our study. In terms of presence of enthesopathy in the knee, we employed a limited definition. We interpreted subspinous central BML but we did not examine enthesopathy of other areas such as at the medial and lateral collateral ligaments. We also selected subjects based on the presence or absence of central BML on knee MRI. Selection of subjects based on the presence or absence of polyarticular hand OA may have identified a subgroup among those with central BML more likely to have hand enthesophytes. It is possible that within our cohort this was an underrepresented subgroup; however, our results did not suggest a greater prevalence of hand enthesopathy among the central BML group, with or without statistical significance. Also, the matter of whether central BML represent permanent findings on knee MRI has not been clearly defined and it is possible that they may exist as transient observations.

We were unable to establish a correlation of hand enthesopathy and knee enthesopathy. Although our investigation was limited to 2 areas of possible enthesopathy, these results may provide evidence against a generalized enthesopathic disorder in association with knee OA.

\section{REFERENCES}

1. Cooper C, Egger P, Coggon D, Hart D, Masud T, Cicuttinit F, et al. Generalized osteoarthritis in women: pattern of joint involvement and approaches to definition for epidemiology studies. J Rheumatol 1996;23:1938-42.

2. Hirsch R, Lethbridge-Cejku M, Scott WW Jr, Reichle R, Plato CC, Tobin J, et al. Association of hand and knee osteoarthritis: Evidence for a polyarticular disease subset. Ann Rheum Dis 1996;55:25-9.

3. Cushnaghan J, Dieppe P. Study of 500 patients with limb joint osteoarthritis. I. Analysis by age, sex, and distribution of symptomatic joint sites. Ann Rheum Dis 1991;50:8-13.

4. Vignon E. Hand osteoarthritis and generalized osteoarthritis: A need for clarification. Osteoarthritis Cartilage 2000;8 Suppl A:S22-4.

5. Slobodin G, Rozenbaum M, Boulman N, Rosner I. Varied presentations of enthesopathy. Semin Arthritis Rheum 2007;37:119-26.

6. Rogers J, Shepstone L, Dieppe P. Is osteoarthritis a systemic disorder of bone? Arthritis Rheum 2004;50:452-7.

7. Rogers J, Shepstone L, Dieppe P. Bone formers: Osteophyte and enthesophyte formation are positively associated. Ann Rheum Dis 1997;56:85-90.

8. Felson DT, Neogi T. Osteoarthritis: Is it a disease of cartilage or of bone? Arthritis Rheum 2004;50:341-4.

9. Tan A, Grainger A, Tanner S, Shelley D, Pease C, Emery P, et al. High-resolution magnetic resonance imaging for the assessment of 
hand osteoarthritis. Arthritis Rheum 2005;52:2355-65.

10. Benjamin M, Moriggl B, Brennan E, Emery P, McGonagle D, Redman S. The "enthesis organ" concept: Why enthesopathies may not present as focal insertional disorders. Arthritis Rheum 2004;50:3306-13.

11. Benjamin M, McGonagle D. Histopathologic changes at "synovio-entheseal complexes" suggesting a novel mechanism for synovitis in osteoarthritis and spondylarthritis. Arthritis Rheum 2007;56:3601-09.

12. Zanetti M, Bruder E, Romero J, Hodler J. Bone marrow edema in osteoarthritic knees: Correlation between MR imaging and histologic findings. Radiology 2000;215:835-40.

13. Hernandez-Molina G, Guermazi A, Niu J, Gale D, Goggins J, Amin $\mathrm{S}$, et al. Central bone marrow lesions in symptomatic knee osteoarthritis and their relationship to anterior cruciate ligament tears and cartilage loss. Arthritis Rheum 2008;58:130-6.

14. Englund M, Guermazi A, Gale D, Felson DT. Incidental meniscal findings on knee MRI in middle aged and elderly persons in the United States. N Engl J Med 2008;359:1108-15.
15. Felson DT, Niu J, McClennan C, Sack B, Aliabadi P, Hunter DJ, et al. Knee buckling: Prevalence, risk factors, and associated limitations in function. Ann Intern Med 2007;147:534-40.

16. Karlson EW, Sanchez-Guerrero J, Wright EA, Lew RA, Daltroy LH, Katz JN, et al. A connective tissue disease screening questionnaire for population studies. Ann Epidemiol 1995;5:297-302.

17. Kellgren JH, Lawrence JS. Radiological assessment of osteoarthrosis. Ann Rheum Dis 1957;16:494-501.

18. Kalichman L, Malkin I, Kobyliansky E. Hand bone midshaft enthesophytes: the influence of age, sex, heritability. Osteoarthritis Cartilage 2007;15:1113-9.

19. Littlejohn GO, Urowitz MB, Smythe HA, Keystone EC. Radiographic features of the hand in diffuse idiopathic skeletal hyperostosis (DISH): Comparison with normal subjects and acromegalic patients. Radiology 1981;140:623-9. 